\title{
m-NIL-CLEAN COMPANION MATRICES
}

\author{
A. CÎMPEAN ${ }^{\dagger}$
}

\begin{abstract}
Companion matrices over fields of positive characteristic, $p$, that are sums of $m$ idempotents, $m \geq 2$, and a nilpotent are characterized in terms of dimension and trace of such a matrix and of $p$.
\end{abstract}

Key words. Companion matrix, Idempotent, Nilpotent, m-nil-clean.

AMS subject classifications. 15A24, 15A83, 16U99.

1. Introduction. In [10], clean rings and clean elements in rings were introduced, in order to study some properties of direct decompositions of modules. Clean elements are sums of a unit and an idempotent element of the ring and a clean ring is such that all of its elements are clean. A particular class of clean rings was introduced by Diesl in [8]: the class of rings such that all elements are sums of a nilpotent and an idempotent. Other generalizations were considered in [6] and [4]. In the former, Chen and Sheibani considered 2-nil-clean rings, rings such that all elements are 2-nil-clean, i.e., elements that are sums of two idempotents and a nilpotent element. Weakly nil-clean rings were firstly introduced in the commutative case by Danchev and McGovern, in [7]. Breaz, Danchev and Zhou characterized weakly nil-clean rings in [4]. In the case of these rings, each element is a sum or a difference of a nilpotent and an idempotent. Moreover, in [1], the author studies elements which are sums of a nilpotent and $\mathrm{m}$ idempotents which commute.

It was proven in [9] that matrix rings over clean rings are clean. Matrix rings over nil-clean rings were studied in [8] and [3]. In the latter, it was proven that a matrix ring over a commutative nil-clean ring is nil-clean. Nil-clean matrices over general fields were studied in [5], where the authors study nil-clean companion matrices. We note that this can be an important step in a possible attempt to characterize general nil-clean matrices since every matrix is similar to a direct sum of companion matrices.

Using this idea we will study in this paper $m$-nil-clean companion matrices over fields of positive characteristic. Let $m \geq 2$ be an integer. Then an $m$-nil-clean element of a ring is an element that represents the sum of $m$ idempotents and a nilpotent (of that ring). In Theorem 3.4, we characterize $m$-nil-clean companion matrices by using the dimension and the trace of such a matrix and the characteristic of the field.

Let $\mathbb{F}$ be a field of positive characteristic, $p$. Let $q$ be a monic polynomial over $\mathbb{F}, q=X^{n}+c_{n-1} X^{n-1}+$ $\cdots+c_{1} X+c_{0}$. The companion matrix associated to $q$ is the $n \times n$ matrix

$$
C=C_{c_{0}, c_{1}, \ldots, c_{n-1}}=\left(\begin{array}{ccccc}
0 & 0 & \cdots & 0 & -c_{0} \\
1 & 0 & \cdots & 0 & -c_{1} \\
\vdots & \vdots & & \vdots & \vdots \\
0 & 0 & \cdots & 1 & -c_{n-1}
\end{array}\right) .
$$

We also denote $C$ by $C_{q}$.

${ }^{*}$ Received by the editors on March 18, 2019. Accepted for publication on October 11, 2019. Handling Editor: Sergey Sergeev.

†Babeş-Bolyai University, Faculty of Mathematics and Computer Science, Str. Mihail Kogălniceanu 1, 400084, Cluj-Napoca, Romania (cimpean_andrada@yahoo.com). 
2. Useful tools. Any matrix is similar to a Frobenius normal form (a direct sum of companion matrices), a matrix similar to a nilpotent is nilpotent and a matrix similar to an idempotent is idempotent. This is why in the proof of Theorem 3 of [2], one restricts without loss of generality to the case of companion matrices. The same technique is used in [6] to prove that $M_{n}\left(\mathbb{F}_{3}\right)$ is 2-nil-clean. We are also determined to consider $m$-nil-clean companion matrices, based on the previously mentioned facts and on the fact that, if all companion matrices which appear in the Frobenius normal form of a matrix $A$ are $m$-nil-clean, then $A$ is also $m$-nil-clean.

Let $\mathbb{F}$ be a field of positive characteristic $p$. In [5], an investigation was made for nil-clean companion matrices over $\mathbb{F}$.

TheOREM 2.1. Let $\mathbb{F}$ be a field of positive characteristic $p$. Let $C=C_{c_{0}, c_{1}, \ldots, c_{n-1}} \in M_{n}(\mathbb{F})$ be a companion matrix. The following are equivalent:

1. $C$ is nil-clean.

2. One of the following conditions is true:

(a) $C$ is nilpotent (i.e., $c_{0}=\cdots=c_{n-1}=0$ );

(b) $C$ is unipotent (i.e., $c_{i}=(-1)^{i}\left(\begin{array}{c}n \\ n-i\end{array}\right)$ for all $i \in\{0, \ldots, n-1\}$ );

(c) there exists an integer $k \in\{1, \ldots, p\}$ such that $-c_{n-1}=k \cdot 1$ and $n>k$.

As a consequence of this fact, the following result holds for characteristic 2:

Corollary 2.2. Let $\mathbb{F}$ be a field of characteristic 2. Let $C=C_{c_{0}, c_{1}, \ldots, c_{n-1}} \in M_{n}(\mathbb{F})$ be a companion matrix. Then $C$ is nil-clean if and only if $-c_{n-1} \in\{0,1\}$.

Here is another corollary of the above theorem:

COROLlary 2.3. Let $n \geq 3$ be a positive integer. The following are equivalent for a field $\mathbb{F}$ :

1. $\mathbb{F} \cong \mathbb{F}_{p}$ for a prime $p<n$;

2. every companion matrix $C \in M_{n}(\mathbb{F})$ is nil-clean.

In [2] and [11], the authors use some decompositions which involve matrices that are the sum of a diagonal matrix with entries only 0 and 1 and a companion matrix. In the following, we will use a similar technique.

The following lemma will be useful while proving results on $m$-nil-clean companion matrices. We will use the notation: $\operatorname{Lin}\left(\left\{v_{1}, v_{2}, \ldots, v_{n}\right\}\right)$ for the subspace of a vector space $X$ generated by the set of vectors $v_{1}, v_{2}, \ldots, v_{n}$ of $X$.

Lemma 2.4. Let $\mathbb{F}$ be a field. For every companion matrix $C_{q} \in M_{n}(\mathbb{F})$ and every $k \in\{1, \ldots, n\}$, there exists a companion matrix $C_{q^{\prime}}$ such that $C_{q}$ and $\operatorname{diag}(\underbrace{1, \ldots, 1}_{k \text {-times }}, 0, \ldots, 0)+C_{q^{\prime}}$ are similar.

Proof. First we will prove the statement for $k \in\{1,2, \ldots, n-1\}$. Let $V$ denote the $n$-dimensional vector space of columns over $\mathbb{F}$ and consider $C_{q}$ as an endomorphism $C_{q}: V \rightarrow V$. Denoting by $\left\{e_{1}, e_{2}, \ldots, e_{n}\right\}$ the standard basis of $V, C_{q}$ maps each $e_{i}$ to $e_{i+1}$, for each $i \in\{1,2, \ldots, n-1\}$.

Now we define $\left\{f_{1}, f_{2}, \ldots, f_{n}\right\}, f_{i} \in V, i \in\{1,2, \ldots, n-1\}$, inductively as it follows. First set $f_{1}=e_{1}$. Assuming that $2 \leq i \leq n$ and that $f_{i-1}$ has been defined, set $f_{i}=C_{q}\left(f_{i-1}\right)-f_{i-1}$, if $i \in\{1,2, \ldots, k+1\}$ and $f_{i}=C_{q}\left(f_{i-1}\right)$, if $i \in\{k+2, \ldots, n\}$ 
We have $e_{1}=f_{1}$, so $e_{1} \in \operatorname{Lin}\left(\left\{f_{1}\right\}\right)$ and $f_{2}=C_{q}\left(f_{1}\right)-f_{1}=C_{q}\left(e_{1}\right)-f_{1}=e_{2}-f_{1}$, so $e_{2}=f_{1}+f_{2}$ and $e_{2} \in \operatorname{Lin}\left(\left\{f_{1}, f_{2}\right\}\right)$

It is easy to see that each $f_{i}$ is the sum of $e_{i}$ and a linear combination of $e_{i-1}, e_{i-2}, \ldots, e_{2}, e_{1}$. Hence, $e_{i}$ is the difference of $f_{i}$ and a linear combination of $e_{i-1}, e_{i-2}, \ldots, e_{2}, e_{1}$. Assuming $e_{1}, e_{2}, \ldots, e_{i-1} \in$ $\operatorname{Lin}\left(\left\{f_{1}, f_{2}, \ldots, f_{n}\right\}\right)$, we get $e_{i}$ is a linear combination of $f_{1}, f_{2}, \ldots, f_{n}$. Therefore, $\operatorname{Lin}\left(\left\{e_{1}, e_{2}, \ldots, e_{n}\right\}\right)=$ $\operatorname{Lin}\left(\left\{f_{1}, f_{2}, \ldots, f_{n}\right\}\right)$, and thus, $\left\{f_{1}, f_{2}, \ldots, f_{n}\right\}$ is a basis of $V$.

Moreover, by the definition, we have:

$$
\begin{gathered}
C_{q}\left(f_{1}\right)=f_{1}+f_{2}, \\
C_{q}\left(f_{2}\right)=f_{2}+f_{3}, \\
\vdots \\
C_{q}\left(f_{k}\right)=f_{k}+f_{k+1}, \\
C_{q}\left(f_{k+1}\right)=f_{k+2}, \\
\vdots \\
C_{q}\left(f_{n-1}\right)=f_{n} .
\end{gathered}
$$

Let $M$ be the matrix the endomorphism $C_{q}$ corresponds to, with respect to the basis $B=\left\{f_{1}, f_{2}, \ldots, f_{n}\right\}$. Therefore,

$$
M=\left[\left[C_{q}\left(f_{1}\right)\right]_{B},\left[C_{q}\left(f_{2}\right)\right]_{B}, \ldots,\left[C_{q}\left(f_{k}\right)\right]_{B},\left[C_{q}\left(f_{k+1}\right)\right]_{B}, \ldots,\left[C_{q}\left(f_{n-1}\right)\right]_{B},\left[C_{q}\left(f_{n}\right)\right]_{B}\right] .
$$

Hence,

$$
M=\left[\left[f_{1}+f_{2}\right]_{B},\left[f_{2}+f_{3}\right]_{B}, \ldots,\left[f_{k}+f_{k+1}\right]_{B},\left[f_{k+2}\right]_{B}, \ldots,\left[f_{n}\right]_{B},\left[C_{q}\left(f_{n}\right)\right]_{B}\right] .
$$

It follows that $M=\operatorname{diag}(\underbrace{1, \ldots, 1}_{k \text {-times }}, 0, \ldots, 0)+C_{q^{\prime}}$ for some monic polynomial $q^{\prime}$ of degree $n$. So, $C_{q}=$ $P^{-1}\left(\operatorname{diag}(\underbrace{1, \ldots, 1}_{k \text {-times }}, 0, \ldots, 0)+C_{q^{\prime}}\right) P$, where $P$ is the transition matrix mapping each $e_{i}$ to $f_{i}$.

As next step, we will solve the case $k=n$, that is we will prove that $C$ is similar to $I_{n}+C_{q^{\prime}}$, where $q^{\prime}=q^{\prime \prime}+X^{n-1}$, and $q^{\prime \prime}$ is such that $\operatorname{diag}(\underbrace{1, \ldots, 1}_{n-1 \text {-times }}, 0)+C_{q^{\prime \prime}}$ is similar to $C$. We consider the vector space of column vectors of dimension $n$ over $\mathbb{F}$. Let $P$ be the transition matrix from canonical basis to basis $\mathrm{B}$ defined in the first part of this lemma, taking $C_{q}=C$. Then $P\left(C_{q}-I_{n}\right) P^{-1}=P C_{q} P^{-1}-I_{n}=$ $\operatorname{diag}(\underbrace{1, \ldots, 1}_{n-1 \text {-times }}, 0)+C_{q^{\prime \prime}}-I_{n}=C_{q^{\prime}}$, where $q^{\prime}=q^{\prime \prime}+X^{n-1}$. Therefore, $C-I_{n}$ is similar to $C_{q^{\prime}}$, where $q^{\prime}=q^{\prime \prime}+X^{n-1}$, and $q^{\prime \prime}$ is such that $\operatorname{diag}(\underbrace{1, \ldots, 1}_{n-1 \text {-times }}, 0)+C_{q^{\prime \prime}}$ is similar to $C$. Hence, $C_{q}$ is similar to $I_{n}+C_{q^{\prime}}$.

EXAmple 2.5. For $p=11, n=6, k=5, q=X^{6}+3 X^{5}-X^{3}-X^{2}-X-1$ we want to see what matrix of the type $\operatorname{diag}(\underbrace{1, \ldots, 1}_{k \text {-times }}, 0, \ldots, 0)+C_{q^{\prime}}$ is similar to $C_{q}$. We want to express the vectors in the basis $B=\left\{f_{1}, f_{2}, f_{3}, f_{4}, f_{5}, f_{6}\right\}$ as linear combinations of vectors in the canonical basis and vectors in the canonical basis as linear combinations of vectors in basis $B$ in order to find out the transition matrix from canonical basis to basis $B$ and its inverse $\left(P\right.$ and $\left.P^{-1}\right)$. 
After doing this we obtain

$$
P=\left(\begin{array}{cccccc}
1 & 1 & 1 & 1 & 1 & 1 \\
0 & 1 & 2 & 3 & 4 & 5 \\
0 & 0 & 1 & 3 & 6 & -1 \\
0 & 0 & 0 & 1 & 4 & -1 \\
0 & 0 & 0 & 0 & 1 & 5 \\
0 & 0 & 0 & 0 & 0 & 1
\end{array}\right) \quad \text { and } \quad P^{-1}=\left(\begin{array}{cccccc}
1 & -1 & 1 & -1 & 1 & -1 \\
0 & 1 & -2 & 3 & -4 & 5 \\
0 & 0 & 1 & -3 & 6 & 1 \\
0 & 0 & 0 & 1 & -4 & -1 \\
0 & 0 & 0 & 0 & 1 & -5 \\
0 & 0 & 0 & 0 & 0 & 1
\end{array}\right)
$$

Since $C_{q}=P^{-1}\left(\operatorname{diag}(1,1,1,1,1,0)+C_{q^{\prime}}\right) P$, we have

$$
C_{q}=\left(\begin{array}{cccccc}
0 & 0 & 0 & 0 & 0 & 1 \\
1 & 0 & 0 & 0 & 0 & 1 \\
0 & 1 & 0 & 0 & 0 & 1 \\
0 & 0 & 1 & 0 & 0 & 1 \\
0 & 0 & 0 & 1 & 0 & 0 \\
0 & 0 & 0 & 0 & 1 & -3
\end{array}\right) \sim\left(\begin{array}{cccccc}
1 & 0 & 0 & 0 & 0 & 0 \\
1 & 1 & 0 & 0 & 0 & 7 \\
0 & 1 & 1 & 0 & 0 & 3 \\
0 & 0 & 1 & 1 & 0 & 6 \\
0 & 0 & 0 & 1 & 1 & 3 \\
0 & 0 & 0 & 0 & 1 & 3
\end{array}\right)=\operatorname{diag}(1,1,1,1,1,0)+C_{q^{\prime}}
$$

3. $m$-nil-clean companion matrices. As a consequence of Theorem 2.1, if $n>p$, then every $n \times n$ companion matrix over $\mathbb{F}$ is nil-clean, where $\mathbb{F}$ is a field of positive characteristic $p$. Therefore, we will assume that $n \leq p$.

Secondly, for $n=1$ the only nilpotent of $M_{n}(\mathbb{F})$ is $(0)$ and the only idempotents of $M_{n}(\mathbb{F})$ are $(0)$ and (1). Therefore, $C \in M_{1}(\mathbb{F})$ is $m$-nil-clean if and only if $C \in\{(0),(1),(2), \ldots,(m)\}$. Hence, we will not refer to the case $n=1$, so we will assume $n>1$ from now on.

LEMma 3.1. Let $m \geq 2$ be an integer. Let $\mathbb{F}$ be a field of positive characteristic $p$. Let $A \in \mathbb{M}_{n}(\mathbb{F})$ be a (not necessarily companion) matrix, for which there exists the decomposition $A=E_{1}+E_{2}+\cdots+E_{m}+N$, with $k_{i}=\operatorname{rank}\left(E_{i}\right), E_{i}$ idempotent, $i \in\{1,2, \ldots, m)$ and $N$ is nilpotent. Then there is an integer $c$ such that $\operatorname{trace}(A)=c \cdot 1$, and $c=k_{1}+k_{2}+\cdots+k_{m}(\bmod p)$ and each $k_{i}$ is a natural number less than or equal to $n$.

Proof. If $A=E_{1}+E_{2}+\cdots+E_{m}+N, E_{i}$ idempotent, $i \in\{1, \ldots, m\}$ and $N$ is nilpotent, then $\operatorname{trace}(A)=\operatorname{trace}\left(E_{1}\right)+\operatorname{trace}\left(E_{2}\right)+\cdots+\operatorname{trace}\left(E_{m}\right)+\operatorname{trace}(N)$. It follows that $\operatorname{trace}(A)=\operatorname{trace}\left(E_{1}\right)+$ $\operatorname{trace}\left(E_{2}\right)+\cdots+\operatorname{trace}\left(E_{m}\right)$. Moreover, it is known that if $E$ is an idempotent, $\operatorname{then} \operatorname{trace}(E)=\operatorname{rank}(E) \cdot 1$. So $\operatorname{trace}(A)=\left(k_{1}+k_{2}+\cdots+k_{m}\right) \cdot 1$, and $k_{i} \leq n, i \in\{1,2, \ldots, m\}$.

LEMMA 3.2. Let $\mathbb{F}$ be a field of positive characteristic $p, 1<n \leq p$. If $-c_{n-1}=c \cdot 1$ and $c \in\{2,3, \ldots, 2 n-$ $2,2 n-1\}$, then $C=C_{c_{0}, c_{1}, \ldots, c_{n-1}} \in M_{n}(\mathbb{F})$ is 2-nil-clean.

Proof. Since $c \in\{2,3, \ldots, 2 n-1\}$, there exist $k \in\{1,2, \ldots, n\}$ and $l \in\{1,2, \ldots, n-1\}$ such that $c \cdot 1=(k+l) \cdot 1$. Let $C=C_{q}$, where $q=X^{n}+c_{n-1} X^{n-1}+\cdots+c_{1} X+c_{0}$. Then, by Lemma 2.4 , there exists $q^{\prime}=X^{n}+d_{n-1} X^{n-1}+\cdots+d_{1} X+d_{0}, q^{\prime} \in \mathbb{F}[X]$ such that

$$
C_{q} \sim \operatorname{diag}(\underbrace{1, \ldots, 1}_{k \text {-times }}, 0, \ldots, 0)+C_{d_{0}, d_{1}, \ldots, d_{n-1}} .
$$

Hence, it follows $-c_{n-1}=k-d_{n-1}$ and so $-d_{n-1}=l \cdot 1$, and we know $n>l$ and $l \neq 0$. Now, by Theorem 2.1, it follows that there exist an idempotent matrix $E$ and a nilpotent matrix $N$ such that $C_{d_{0}, d_{1}, \ldots, d_{n-1}}=E+N$. Using this and equation 3.1 we have $C_{q} \sim \operatorname{diag}(1, \ldots, 1,0, \ldots, 0)+E+N$. We know 
that $C_{q}=P^{-1}\left(\operatorname{diag}(\underbrace{1, \ldots, 1}_{k \text {-times }}, 0, \ldots, 0)+C_{d_{0}, d_{1}, \ldots, d_{n-1}}\right) P$, where $P$ is the transition matrix mapping each $e_{i}$ of canonical basis to $f_{i}$ of the basis met in Lemma 2.4. Now we have

$$
C_{q}=P^{-1} \operatorname{diag}(1, \ldots, 1,0, \ldots, 0) P+P^{-1} E P+P^{-1} N P .
$$

Since a matrix similar to an idempotent is an idempotent and a matrix similar to a nilpotent is nilpotent, we have actually showed the fact that $C_{q}$ is 2-nil-clean.

LEMma 3.3. Let $m \geq 2$ be an integer. Let $\mathbb{F}$ be a field of positive characteristic $p$, and $1<n \leq p$. If $-c_{n-1}=c \cdot 1$ and $c \in\{m, m+1, \ldots, m n-1\}$, then $C=C_{c_{0}, c_{1}, \ldots, c_{n-1}} \in \mathbb{M}_{n}(\mathbb{F})$ is m-nil-clean.

Proof. This lemma's statement is proved for $m=2$ in Lemma 3.2.

Now assume that the lemma's statement is true for $m \geq 2$. We will prove that it is true also for $m+1$. Let $c \in\{m+1, m+2, \ldots,(m+1) n-1\}$. We will prove that $C$ is $(m+1)$-nil-clean.

Since $c \in\{m+1, m+2, \ldots,(m+1) n-1\}$, it follows that there exist $k \in\{1,2, \ldots, n\}$ and $l \in$ $\{m, m+1, \ldots, m n-1\}$ such that $c \cdot 1=(k+l) \cdot 1$. Let $C=C_{q}, q=X^{n}+c_{n-1} X^{n-1}+\cdots+c_{1} X+c_{0}$. Then by Lemma 2.4 , there exists $q^{\prime}=X^{n}+d_{n-1} X^{n-1}+\cdots+d_{1} X+d_{0}, q^{\prime} \in \mathbb{F}[X]$, such that

$$
C_{q} \sim \operatorname{diag}(\underbrace{1, \ldots, 1}_{k \text {-times }}, 0, \ldots, 0)+C_{d_{0}, d_{1}, \ldots, d_{n-1}} .
$$

It follows $-c_{n-1}=k-d_{n-1}$ and so $-d_{n-1}=l \cdot 1$. We know that $l \in\{m, m+1, \ldots, m n-1\}$, so by hypothesis induction we obtain that there exist idempotent matrices $E_{1}, E_{2}, \ldots, E_{m}$, and the nilpotent matrix $N$, such that $C_{d_{0}, d_{1}, \ldots, d_{n-1}}=E_{1}+E_{2}+\cdots+E_{m}+N$. Using this and equation 3.3 we have

$$
C_{q} \sim \operatorname{diag}(\underbrace{1, \ldots, 1}_{k \text {-times }}, 0, \ldots, 0)+E_{1}+E_{2}+\cdots+E_{m}+N .
$$

We know that there exists an invertible matrix $P$ such that

$$
C_{q}=P^{-1}\left(\operatorname{diag}(\underbrace{1, \ldots, 1}_{k \text {-times }}, 0, \ldots, 0)+C_{d_{0}, d_{1}, \ldots, d_{n-1}}\right) P .
$$

Therefore,

$$
C_{q}=P^{-1}(\operatorname{diag}(\underbrace{1, \ldots, 1}_{k \text {-times }}, 0, \ldots, 0)) P+P^{-1} E_{1} P+P^{-1} E_{2} P+\cdots+P^{-1} E_{m} P+P^{-1} N P .
$$

Since a matrix similar to an idempotent is idempotent and a matrix similar to a nilpotent is nilpotent, we have actually showed the fact that $C_{q}$ is $(m+1)$-nil-clean.

Having the case $p=2$ solved in Corollary 2.2 ( $C$ is nil-clean in this case, so is also $m$-nil-clean), we can assume from now on that $p$ is odd.

THEOREM 3.4. Let $m \geq 2$ be an integer. Let $\mathbb{F}$ be a field of positive odd characteristic $p$, and $1<n \leq p$. Let $C=C_{c_{0}, c_{1}, \ldots, c_{n-1}}$ be a companion matrix. Let c be the smallest nonnegative integer such that $-c_{n-1}=c \cdot 1$.

The following hold:

1. If $c=0$ and $m n-1<p$, then $C$ is m-nil-clean if and only if $C$ is nilpotent or $C-(m-1) I_{n}$ is unipotent. 
2. If $c=t, 1 \leq t \leq m$, then $C$ is $t$-nil-clean (1-nil-clean is just nil-clean), so is m-nil-clean.

3. If $c \in\{m, m+1, \ldots, m n-2, m n-1\}$, then $C$ is m-nil-clean.

4. If $m n-2 \geq p$, then $C$ is m-nil-clean.

5. Assume that $m n-2<p$.

(a) If $c=m n$ and $p=m n-1$, then $C$ is nil-clean, so is m-nil-clean.

(b) If $c=m n$ and $p=m n$, then $C$ is $m$-nil-clean if and only if $C$ is nilpotent or $C-(m-1) I_{n}$ is an unipotent matrix.

(c) If $c=m n, p>m n$, then $C$ is m-nil-clean if and only if $C-(m-1) I_{n}$ is an unipotent matrix.

(d) If $c>m n$, then $C$ is not m-nil-clean.

Proof.

1. If $c=0$ and $m n-1<p$, then $c=0$ and $m n \leq p$.

If $m n<p$, then $c=(\underbrace{0+\cdots+0}_{m \text {-times }})$ is the only form of $c$ as sum, modulo $p$ of $m$ positive integers less or equal to $n$, and since $\mathrm{C}$ is $m$-nil-clean it follows that the idempotents in the $m$-nil-clean decomposition of $C$ have rank zero. Therefore, they are $O_{n}$, and hence, $C$ is nilpotent.

If $m n=p$, then $c=(\underbrace{0+\cdots+0}_{m \text {-times }})$ and $c=(\underbrace{n+\cdots+n}_{m \text {-times }})$ are the only forms of $c$ as a sum modulo $p$ of $m$ positive integers less or equal to $n$, and, since $C$ is $m$-nil-clean, it follows $C$ is nilpotent or $C-(m-1) I_{n}$ is unipotent.

It is obvious that if $C$ is nilpotent or $C-(m-1) I_{n}$ is unipotent, then $C$ is $m$-nil-clean.

2. If $c=1$ and $n>1$, then we have by Theorem 2.1 that $C$ is nil-clean. If $c=t, 2 \leq t \leq m$, by Lemma 3.3 , we have that $C$ is $t$-nil-clean.

3. This is Lemma 3.3.

4. This is a direct consequence of 3 .

5. (a) If $c=m n$ and $p=m n-1$, it follows that $-c_{n-1}=1 \cdot 1$, so $C$ is nil-clean.

(b) Since $c=m n, p=m n$ it follows that $c=0$ and $m n-1<p$, so by 1 . we have that $C$ is $m$-nil-clean if and only if $\mathrm{C}$ is nilpotent or $C-(m-1) I_{n}$ is unipotent.

(c) Since $c=m n, p>m n$, it follows that $-c_{n-1}=m n=(\underbrace{n+n+\cdots+n}_{m \text {-times }})(\bmod p)$, that is the only decomposition as a sum of $m$ integers between 0 and $n$. Hence, the idempotents in the $m$-nil-clean decomposition of $C$ are idempotent units, which means they are $I_{n}$, so $C-(m-1) I_{n}$ is unipotent. Conversely, if $C-(m-1) I_{n}$ is unipotent, then it is obvious that $C$ is $m$-nil-clean.

(d) If $c>m n$, then $c$ cannot be written modulo $p$ as sum of $m$ integers less or equal to $n$, but by Lemma 3.1, we have that $C$ is not $m$-nil-clean.

REMARK 3.5. In Theorem 3.4, one of the conclusions was that $C-(m-1) I_{n}$ is unipotent. We can prove that $C-(m-1) I_{n}$ is similar to a companion matrix, so one can say more, the companion matrix with whom $C-(m-1) I_{n}$ is similar can be only the unipotent companion matrix of type $n \times n$.

Let us prove that $C-(m-1) I_{n}$ is similar to a companion matrix. To begin with, we have by Lemma 2.4 , case $k=n$, that there exists the monic polynomial $q_{1}$ of degree $\mathrm{n}$ such that $C-I_{n} \sim C_{q_{1}}$. But using again Lemma 2.4, case $k=n$, we get $C_{q_{1}}-I_{n} \sim C_{q_{2}}$. Therefore, one can obtain $C-2 I_{n} \sim C_{q_{2}}$. Repeating these steps we find that there exists the sequence of monic polynomials $\left(q_{i}\right)_{1 \leq i \leq m-1}$ such that $C-i I_{n} \sim C_{q_{i}}$, for $i \in\{1,2, \ldots, m-1\}$. 


\section{REFERENCES}

[1] A.N. Abyzov. Strongly q-nil-clean rings. Siberian Mathematical Journal, 60:197-208, 2019.

[2] S. Breaz. Matrices over finite fields as sums of periodic and nilpotent elements. Linear Algebra and its Applications, 555:92-97, 2018.

[3] S. Breaz, G. Călugăreanu, P. Danchev, and T. Micu. Nil-clean matrix rings. Linear Algebra and its Applications, 439:31153119, 2013.

[4] S. Breaz, P. Danchev, and Y. Zhou. Rings in which every element is either a sum or a difference of a nilpotent and an idempotent. Journal of Algebra and its Applications, 15:1650148, 2016.

[5] S. Breaz and G.C. Modoi. Nil clean companion matrices. Linear Algebra and its Applications, 489:50-60, 2016.

[6] H. Chen and M. Sheibani. Strongly 2-nil-clean rings. Journal of Algebra and its Applications, 16:1750178, 2017.

[7] P.V. Danchev and W.Wm. McGovern. Commutative weakly nil clean unital rings. Journal of Algebra, 425:410-422, 2015.

[8] A.J. Diesl. Nil-clean rings. Journal of Algebra, 383:197-211, 2013.

[9] J. Han and W.K. Nicholson. Extensions of clean rings. Communications in Algebra, 29:2589-2595, 2001.

[10] W.K. Nicholson. Lifting idempotents and exchange rings. Transactions of the American Mathematical Society, 229:269$278,1977$.

[11] J. Šter. On expressing matrices over $\mathbb{Z}_{2}$ as the sum of an idempotent and a nilpotent. Linear Algebra and its Applications, 544:339-349, 2018. 